\title{
Typical Presentation of Stress Associated Vincent Angina - A Case Report
}

\author{
Richa S Gautam, Akhil K Padmanabhan*, Esha Yadav and Prabhuji MLV \\ Department of Periodontology, Krishnadevaraya College of Dental Sciences and Hospital, India
}

*Corresponding author: Akhil K Padmanabhan, Department of Periodontology, Krishnadevaraya College of Dental Sciences and Hospital, India

\begin{abstract}
Necrotizing periodontal diseases are the most severe rapidly destructive, non-communicable, periodontal infection of complex etiology. The diagnosis is based on clinical and radiological features. Necrotizing periodontal lesions are confined to periodontal tissues, including gingiva, periodontal ligament, and alveolar bone. The pathognomonic clinical characteristics are the typical punched out appearance, interproximal craters and spontaneous bleeding. The predisposing factors include host factors, such as psychological stress, immunosuppression, a smoking habit, and poor oral hygiene. If it is left untreated, it may spread laterally and apically to involve the entire gingival complex. In this case report, we presented a 24- year old male with necrotizing gingivitis and no systemic disease with a history of intense stress. The case report describes the clinical diagnosis of Necrotizing ulcerative periodontitis (NUP) and its therapeutic management by conservative oral treatment and regular psychological follow up.
\end{abstract}

Keywords: Necrotizing periodontal disease; Diagnosis; Treatment

Abbreviations: NUP: Necrotizing ulcerative periodontitis; NPD: Necrotizing periodontal diseases; NP: Necrotizing Periodontitis

\section{Introduction}

Necrotizing periodontal diseases (NPD) are the most severe inflammatory periodontal disorders associated with bacterial plaque. It usually has a rapid, aggressive onset, a multifactorial, complex etiology and runs an acute course [1]. They are classified as necrotizing gingivitis, periodontitis, or stomatitis according to the severity of progression of disease and tissue involvement [2]. Classical clinical features in necrotizing periodontitis (NP) are characterized by the presence of punched out, ulcerated and necrotic lesions that may be covered by a pseudo membrane of necrotic tissue [3]. The ulcerations are extremely painful and show spontaneous bleeding. An important feature of NP is the rapid and severe loss of clinical attachment and alveolar bone within a few days or weeks. Secondary clinical features include the presence of fever, oral halitosis, adenopathies and/or general discomfort [3]. This form of gingivitis is relatively rare. A heterogenous set of proliferating oral anaerobic bacteria such as spirochetes, fusobacteria, and strains of Treponema pallidum and Bacteroides intermedius are involved in the development of the clinical signs and symptoms of the disease.1 Primary risk factors for disease include smoking, psychological stress, poor nutrition. Defects in leukocytes and immune function may also be associated with disease occurrence [2]. Here, we report a case of a necrotizing ulcerative gingivitis in a 24 year old male, describing the clinical features, treatment approach, and successful outcomes.

\section{Case Report}

\section{Patient information}

A 24-year-old male patient was referred to the Clinical Department of Periodontology, at the Krishnadevaraya College of dental sciences and hospital Bangalore, with chief complaint of intense and persistent gingival pain, bleeding and foul breath since a year which aggravated in the past two weeks. He weighed $60 \mathrm{~kg}$ and was $182 \mathrm{~cm}$ tall and is a dance artist by profession. The patient gives a history of intense stress for the past few months, improper intake of nutrition and intense stress. The patient also reports elevated body temperature since the past two days and no 
history of drug allergies, current medications, or significant health problems. Presence or absence of Vincent's angina symptoms is

Table 1: Symptoms of Disease in the Patient. mentioned in Table 1. Also, Table 2 shows predisposing factors of the disease.

\begin{tabular}{|c|c|}
\hline Symptoms & Presence or Absence of Disease \\
\hline Inflammation of oropharyngeal mucous membrane & + \\
\hline Redness and bleeding & + \\
\hline Tonsillitis & + \\
\hline Mild to severe pain & + \\
\hline Halitosis & + \\
\hline Anorexia & + \\
\hline Fatigue & + \\
\hline Fever & + \\
\hline
\end{tabular}

+: positive, - : negative.

Table 2: Evaluation of Predisposing Factors in Index Case.

\begin{tabular}{|c|c|}
\hline Risk Factors & - \\
\hline Allergy to food or medicines & - \\
\hline Smoking & - \\
\hline Alcohol & + \\
\hline Poor oral hygiene & + \\
\hline Emotional stress & + \\
\hline Anxiety & - \\
\hline Artificial teeth & - \\
\hline Sensitivity to mouthwashes or tooth pastes & - \\
\hline Radiotherapy or chemotherapy & - \\
\hline Systemic conditions & + \\
\hline Malnutrition & \\
\hline
\end{tabular}

+: positive, - : negative.

\section{Clinical and Diagnostic Findings}

\section{Extra-oral examination}

On extra oral examination, there was no gross facial asymmetry detected, lips were competent, bilateral submandibular lymph nodes were tender on palpation and local rise in body temperature was detected.

\section{Intra-oral examination}

Clinical findings: On intraoral examination, poor oral hygiene
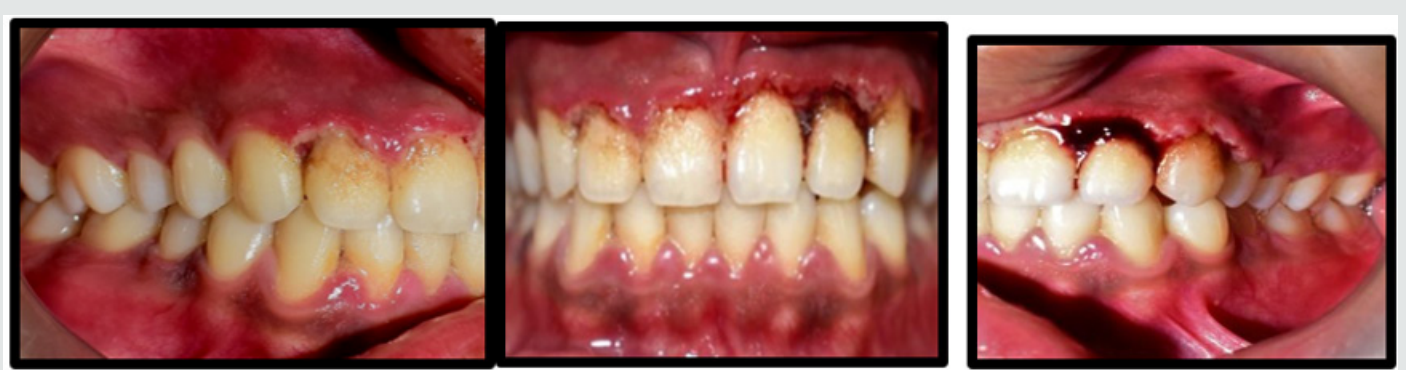

Figure 1: Baseline photograph showing the necrotizing lesion and aberrant frenum.

was recorded with plaque, heavy calculus deposits, visible suppuration associated with fetor oris. The oral lesions were extremely tender hindering periodontal probing. Examination of the gingiva revealed a thin pseudo-membrane that covered a part of the attached gingiva with spontaneous bleeding or on slight provocation. The typical multiple punched out like depressions were found more in interdental papilla and marginal gingiva. The ulcers were found to be more severe and painful in maxilla. The patient had an aberrant labial frenum in the maxilla which added to the severity of lesion in maxillary anterior teeth (Figure 1). 
Radiographic findings: A periapical radiographic examination showed crestal alveolar bone loss in the affected area (Figure 2).

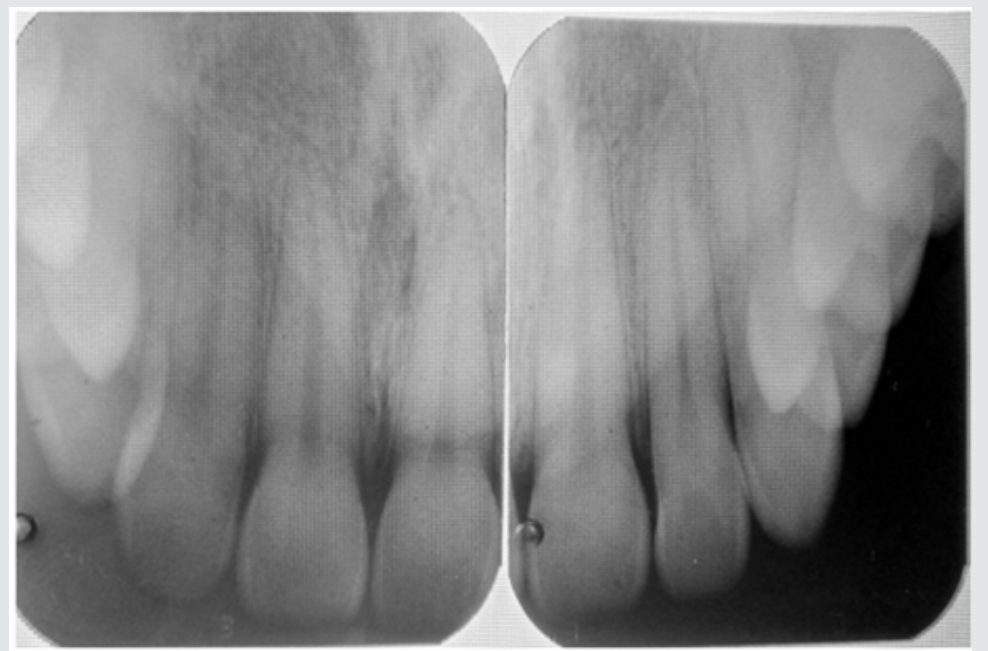

Figure 2: Radiograph showing minimal alveolar bone loss.

Lab investigations: Since necrotising periodontal diseases are most commonly manifested in HIV, biochemical blood analysis and HIV serology were requested. The results of the biochemical blood analysis were normal and the HIV sero-status was negative.

\section{Therapeutic Interventions}

\section{First Visit:}

In the first visit conservative treatment like removal of local factors and maintenance of oral hygiene was done (Figure 3). Removal of the necrotic slough was done under local anaesthesia by using small gauze pellets dipped in $3 \% \mathrm{H}_{2} \mathrm{O}_{2}$. This procedure was done in all the affected areas of the mouth and then the areas were irrigated with saline to remove the unattached debris. Supragingival scaling couldn't be done with ultrasonic scaler as the patient was having discomfort. He was instructed to use a soft brush, hydrogen peroxide 3\% mouth rinse with sterile warm water (1:1) four times a day and chlorhexidine $0.2 \%$ mouthwash twice daily. The patient was prescribed amoxicillin and metronidazole $500 \mathrm{mg}$ tablets three times per day for 5 days, an analgesic, vitamin complexes and a follow up appointment was scheduled. The Patient was recalled after 3 days for evaluation.

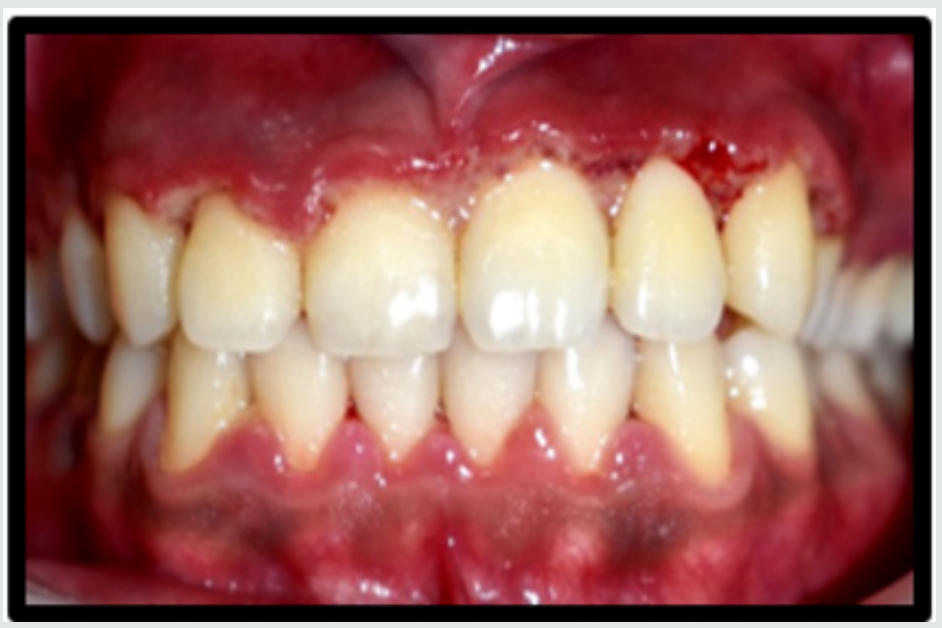

Figure 3: Post scaling and irrigation.

\section{Third visit:}

On the third day of the recall visit a drastic improvement was observed. The ulcers were showing signs of healing, no bleeding points were detected, pain had reduced, and necrotic slough had decreased considerably. The ulcers were still found in the tip of the interdental papilla, inflammatory enlargement was observed
(Figure 4). Lymph nodes were palpable but non tender and the temperature was also normal. Complete scaling was done including subgingival areas to remove local deposits and the ulcerated sites were irrigated with saline. He was instructed to discontinue the hydrogen peroxide mouth rinse and continue the chlorhexidine mouthwash till the next visit which was planned after 4 days. 

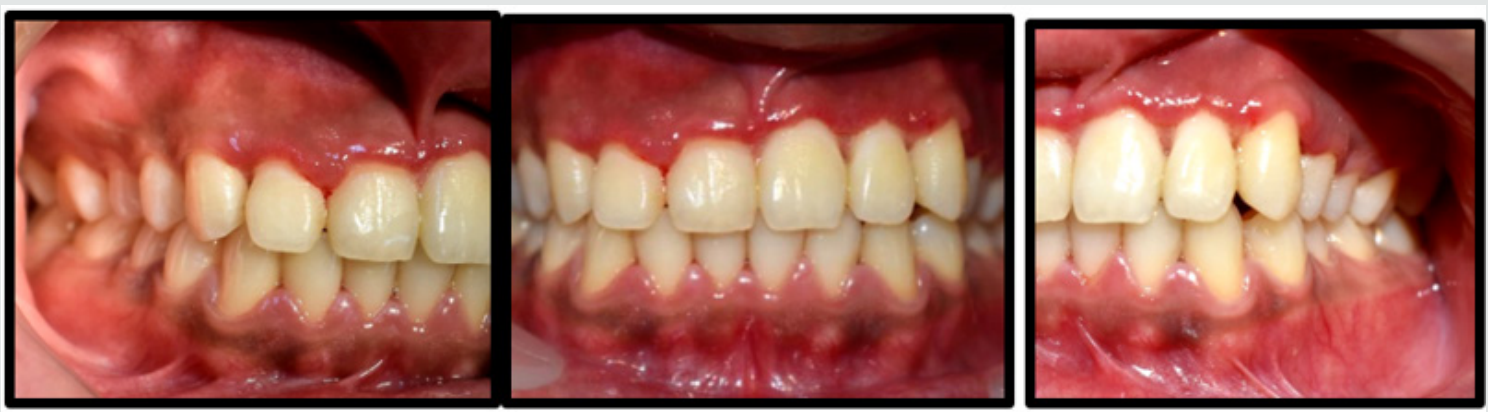

Figure 4: Third day follow up showing moderate inflammation which is resolving.

\section{At Seventh day}

After seven days the patient was almost symptom free. The ulcers had healed and necrotic slough was completely absent. Slight inflammatory enlargement was seen in the maxillary anterior labial and tip of interdental papilla (Figure 5). Oral hygiene instructions were reinforced with prescription of interdental brushes and dental floss to perform mechanical plaque control. Additionally, he was advised to use Chlorhexidine mouth rinse $0.12 \%$ twice a day for further 10 days. Patient was referred to a psychologist to rule out his stress due to his profession. Patient was advised to maintain oral health by regular dental visits and strict oral hygiene practice.

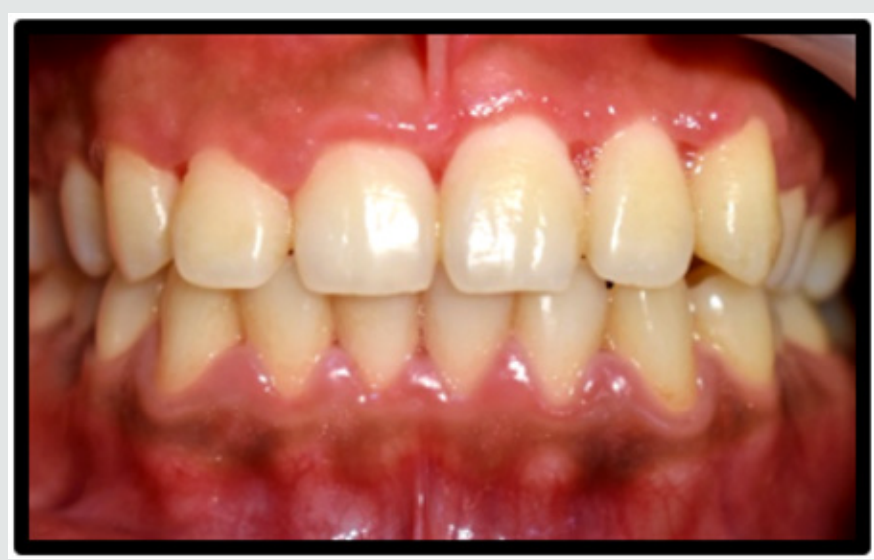

Figure 5: One week follow up showing minimal residual inflammation.

\section{Maintenance phase}

After the completion of the cause-related therapy phase, the patient was enrolled in a periodontal maintenance program to optimize the therapeutic interventions which was done by the patient's adherence to the recall system of weekly followup appointments. Monthly follow-up for the first 3 months post treatment were done and at each visit, the oral hygiene instructions were emphasized.

\section{Clinical outcomes}

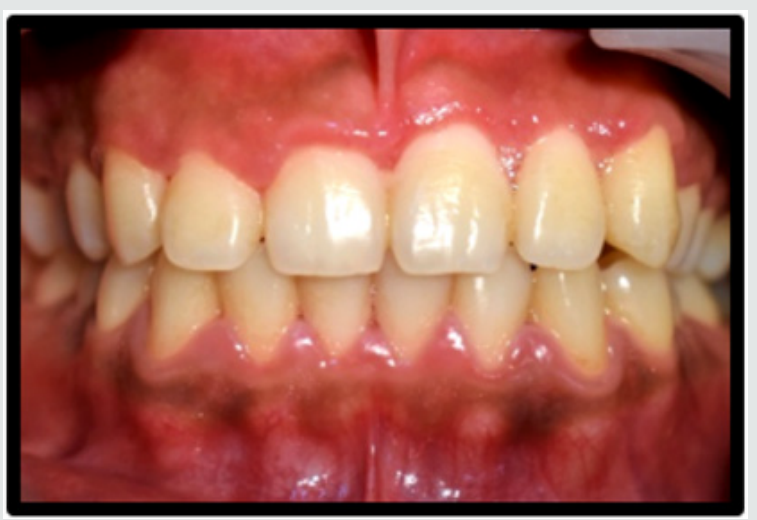

Figure 6: Three months follow up showing completely healed lesion. 
The patient was very compliant and responsive to the treatment provided. At three month recall visit, there was a complete resolution of inflammation and a healing of ulcerated areas with epithelialization of gingival interdental craters (Figure 6). A psychologist was consulted, and the patient was kept on maintenance with instructions of oral hygiene and proper nutrition. The findings at multiple follow-up examinations showed that periodontal health and function were successfully re-established and there was no evidence of progressive attachment loss (Figure $6)$.

\section{Discussion}

Necrotizing periodontitis (NP) is a necrotizing periodontal disease that has an acute onset and requires urgent treatment [1]. The pathognomonic clinical characteristics are the typical punched out appearance and interproximal craters which were evident in our patient. Although its clinical signs are characteristic, the etiology and pathogenesis is still not clear. It is a known fact that the pathogenic processes involved in any periodontal diseases is initiated by bacterial plaque and modified by predisposing factors [2-4]. The bacterial species like fusiform bacilli and spirochetes are dominated in necrotizing periodontal diseases. However, it is not clearly established whether this flora is the cause because of their capacity to invade the epithelium and the connective tissue where they release endotoxins or the consequence of the disease and then they may result from a secondary growth since the necrotic tissues are the perfect environment for bacterial colonization and tissue invasion [5]. Predisposing systemic factors such as AIDS, diabetes, chemotherapy or leukemia are described in the literature to be associated with most cases of NUP [6-8]. In our patient a systemic disease was ruled out since biological analysis was normal and he was otherwise healthy.

This case report describes an acute presentation of necrotizing gingivitis associated with poor oral hygiene, malnutrition, and lack of sleep which are all related to intense psychological stress. Many pathways have been suggested to explain study evaluated the association between levels of the stress-related steroid hormones and periodontitis and reported that high serum levels of cortisol were associated with periodontitis severity [9]. Host immune response to periodontal pathogens may be reduced by increasing the adrenocortical activity, which lead to altered cytokine profile and affected cells recruitment of macrophages and fibroblasts [10]. Stress also causes reduction of tissue matrix metalloproteinase levels, which leads to impaired tissue turnover. During stress periods, the subject's behavior also change and can lead patients to neglect oral hygiene. Malnutrition related to extreme stress has also been reported as a predisposing factor for necrotizing periodontal diseases. The basis for this interaction has been termed 'proteinenergy malnutrition', implying a marked reduction in antioxidant nutrients and an altered acute-phase response against infection [5]. The management strategy for NP lesions were followed in two phases: acute and maintenance phase treatment. The acute phase dealt with removal of local irritating factors. The patient's positive response to the intervention within one week could be attributed to several factors: use of systemic antibiotics like metronidazole, which has been described as the first antibiotic choice because it is active against strict anaerobes, proper oral hygiene, use of vitamin complexes and adequate rest.

\section{Conclusion}

Necrotizing ulcerative lesions, although uncommon, can cause severe pain and discomfort to the patient. It is always associated with underlying systemic illness and hence thorough history recording and clinical examination becomes the key to successful diagnosis and treatment.

\section{References}

1. Holmstrup P (2015) Necrotizing periodontal disease. Lindhe J, Lang NP (Eds.), Clinical periodontology and implant dentistry. $6^{\text {th }}$ ed, WileyBlackwell, Oxford, USA.

2. Horning GM, Cohen ME (1995) Necrotizing ulcerative gingivitis, periodontitis, and stomatitis: clinical staging and predisposing factors. J Periodontol 66: 990-998.

3. Corbet EF (2004) Diagnosis of acute periodontal lesions. Periodontol $200034: 204-216$.

4. Ishikawa I (2007) Host responses in periodontal diseases: a preview. Periodontol 2000 43: 9-13.

5. Herrera D, Alonso B, de Arriba L, Santa Cruz I, Serrano C, et al. (2014) Acute periodontal lesions. Periodontol 2000 65: 149-177.

6. Williams CA, Winkler JR, Grassi M, Murray PA (1990) HIV-associated periodontitis complicated by necrotizing stomatitis. Oral Surg Oral Med Oral Pathol 69: 351-355.

7. Santos FA, Pochapski MT, Pilatti GL, Kozlowski VA, Goiris FA, et al. (2009) Severe necrotizing stomatitis and osteomyelitis after chemotherapy for acute leukaemia. Aust Dent J 54: 262-265.

8. Nakano H, Ota Y, Yura Y (2009) Calcifying epithelial odontogenic tumor of the maxilla with ulcerative stomatitis: a case report. Br J Oral Maxillofac Surg 47: 222-224.

9. Ishisaka A, Ansai T, Soh I, Awano S, Yoshida A, et al. (2008) Association of cortisol and dehydroepiandrosterone sulfate levels in serum with periodontal status in older Japanese adults. J Periodontol 78: 17671773.

10. Deinzer R, Kottmann W, Förster P, Herforth A, Stiller-Winkler R, et al. (2000) After-effects of stress on crevicular interleukin-1beta. J Clin Periodontol 27: 74-77. 
CC (P) This work is licensed under Creative

To Submit Your Article Click Here: $\quad$ Submit Article

DOI: $10.32474 /$ SJ0.2020.04.000190

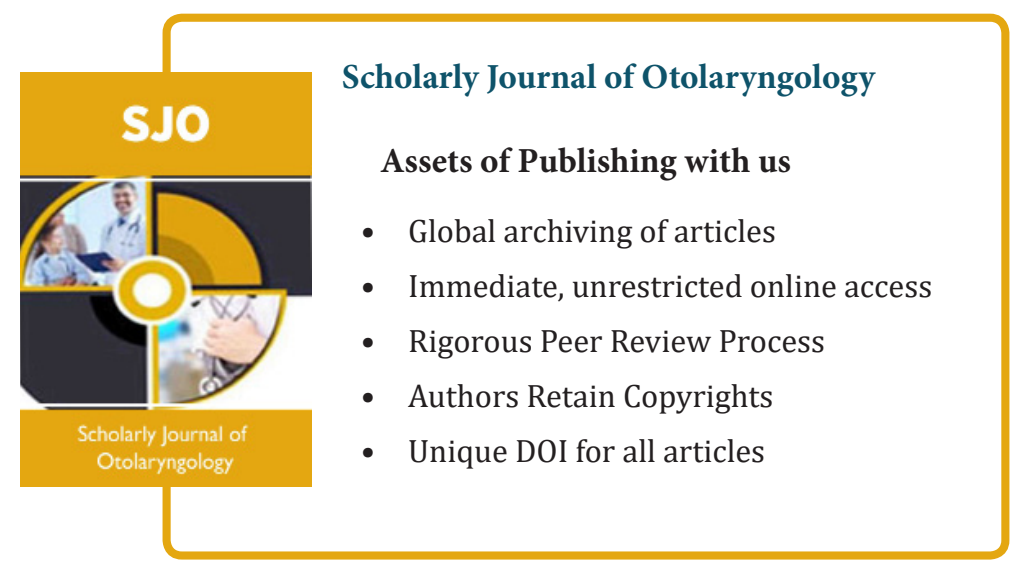

